In conclusion, primary pulmonary lymphatic disorders or neoplasms are rare and are often difficult to diagnose and classify [2]. Lymphatic abnormalities frequently mimic other pathologic processes, particularly neoplastic processes. These lesions are benign (as malignant change has not been documented) but tend to infiltrate surrounding tissues [8]. Provided that the tumour is completely resected, recurrences are rare. We believe this case adds a significant contribution to the literature due to its rarity and unusual location.

Nataliya Khobta*, Pascale Tomasini ${ }^{*, \#}$, Delphine Trousse ${ }^{\#,}$, Fabien Maldonado ${ }^{+}$, Pascal Chanez ${ }^{\#, \S}$ and Philippe Astoul ${ }^{\#, f}$ *Dept of Multidisciplinary Oncology and Therapeutic Innovations, Assistance Publique - Hôpitaux de Marseille, Hôpital Nord, "Dept of Thoracic Surgery, Assistance Publique - Hôpitaux de Marseille, Hôpital Nord, ${ }^{\S}$ Dept of Ambulatory Platforms in Pneumology, Assistance Publique - Hôpitaux de Marseille, Hôpital Nord, ${ }^{f}$ Dept of Thoracic Oncology, Pleural Diseases and Interventional Pulmonology, Assistance Publique - Hôpitaux de Marseille, Hôpital Nord, and "Aix-Marseille Université, Marseilles, France. ${ }^{+}$Division of Pulmonary and Critical Care Medicine, Mayo Clinic, Rochester, MN, USA.

Correspondence: N. Khobta, Dept of Multidisciplinary Oncology and Therapeutic Innovations, Hôpital Nord, Université de la Méditerranée, Chemin des Bourrely, 13915 Marseille Cedex 20, France. E-mail: nataliya.khobta@ap-hm.fr

Statement of Interest: P. Chanez has provided consultancy services for Almirall, Boehringer Ingelheim, Centocor, GlaxoSmithKline, Merck Sharp \& Dohme, AstraZeneca, Novartis, Teva, Chiesi and Schering Plough; has served on advisory boards for Almirall, Boehringer Ingelheim, Centocor, GlaxoSmithKline, AstraZeneca, Novartis, Teva, Chiesi, Schering Plough and Merck Sharp \& Dohme; has received lecture fees from Almirall, Boehringer Ingelheim, Centocor, GlaxoSmithKline, AstraZeneca, Novartis, Teva, Chiesi, Schering Plough and Merck Sharp \& Dohme; and has received industry-sponsored grants from Almirall, Boehringer Ingelheim, Centocor, GlaxoSmithKline, AstraZeneca, Novartis, Teva, Chiesi and Schering Plough.
Acknowledgments: The authors would like to acknowledge M-J. Payan (Hôpital Nord, Marseilles, France) for her expert interpretation of the histopathology specimens.

\section{REFERENCES}

1 Minato H, Kaji S, Kinoshita E, et al. Solitary intrapulmonary cystic lymphangioma in an infant: a case report with literature review. Pathol Res Pract 2006; 2010: 851-856.

2 Green NA, Diaz MC. Pulmonary lymphangioma in a 14-monthold. Pediatr Emerg Care 2011; 27: 52-54.

3 Riquet M, Briere J, Le Pimpec-Barthes F, et al. Les lymphangiomes kystiques du cou et du médiastin: existe-t-il des formes acquises? A propos de 37 cas [Cystic lymphangioma of the neck and mediastinum: are there acquired forms? Report of 37 cases]. Rev Mal Respir 1999; 16: 71-79.

4 Boateng P, Anjum W, Wechsler AS. Vascular lesions of the mediastinum. Thorac Surg Clin 2009; 19: 91-105.

5 Torcasio A, Veneroso S, Amabile MI, et al. Cystic hygroma of the breast: a rare lesion. Tumori 2006; 92: 347-345.

6 Zakaria RH, Barsoum NR, El-Basmy AA, et al. Imaging of pericardial lymphangioma. Ann Pediatr Cardiol 2011; 4: 65-67.

7 Cheng LH, Wells FC. A multidisciplinary approach to recurrent cervico-thoracic cystichygroma in an adult. $\mathrm{Br} J$ Oral Maxillofac Surg 2004; 42: 66-68.

8 Weissferdt A, Moran CA. Primary vascular tumors of the lungs: a review. Ann Diagn Pathol 2010; 14: 296-308.

9 Benninghoff MG, Todd WU, Bascom R.; Incidental pleural-based pulmonary lymphangioma. J Am Osteopath Assoc 2008; 108: 525-528.

10 Balakrishnan A, Bailey CM. Lymphagioma of the tongue. A review of pathogenesis, treatment and the use of surface laser photocoagulation. J Laryngol Otol 1991; 105: 924-929.

11 Copcland J, Müller KM, Müller AM. Pulmonary haemangiolymphangioma - a new entity of pulmonary vascular tumours? Histopathology 2008; 52: 527-529.

12 Nakajima J, Goto A, Takamoto S, et al. Invasive lymphangioma of the lung manifesting as a large pulmonary mass with hemoptysis: report of a case. Surg Today 2007; 37: 418-422.

13 Celikten A, Melek H, Citak N, et al. Minimally invasive excision of multiple cystic lymphangiomas of the mediastinum: a case report. Thorac Cardiovasc Surg 2010; 58: 498-500.

14 Limmer S, Krokowski M, Kujath P. Pulmonary lymphangioma. Ann Thorac Surg 2008; 85: 336-339.

15 Singh O, Singh Gupta S, Upadhyaya VD, et al. Cystic lymphangioma of the breast in a 6-year-old boy. J Pediatr Surg 2009; 44: 2015-2018.

\title{
Pulmonary toxicity after long-term treatment with lenalidomide in two myeloma patients
}

\section{To the Editor:}

A white male was diagnosed with immunoglobulin $G$ k-chain multiple myeloma at age 63 yrs. He initially received a combination of thalidomide, doxorubicin and dexamethasone with concomitant radiotherapy (30 Gy) to the dorsal vertebrae. The first course of this combination was complicated by profound pancytopenia and lobar pneumonia with severe sepsis in the setting of neutropenia, leading to the discontinuation of thalidomide because of concerns regarding haematological toxicity. The pneumonia was not microbiologically documented but considered as being probably of bacterial origin given the clinicoradiological presentation and a satisfying 


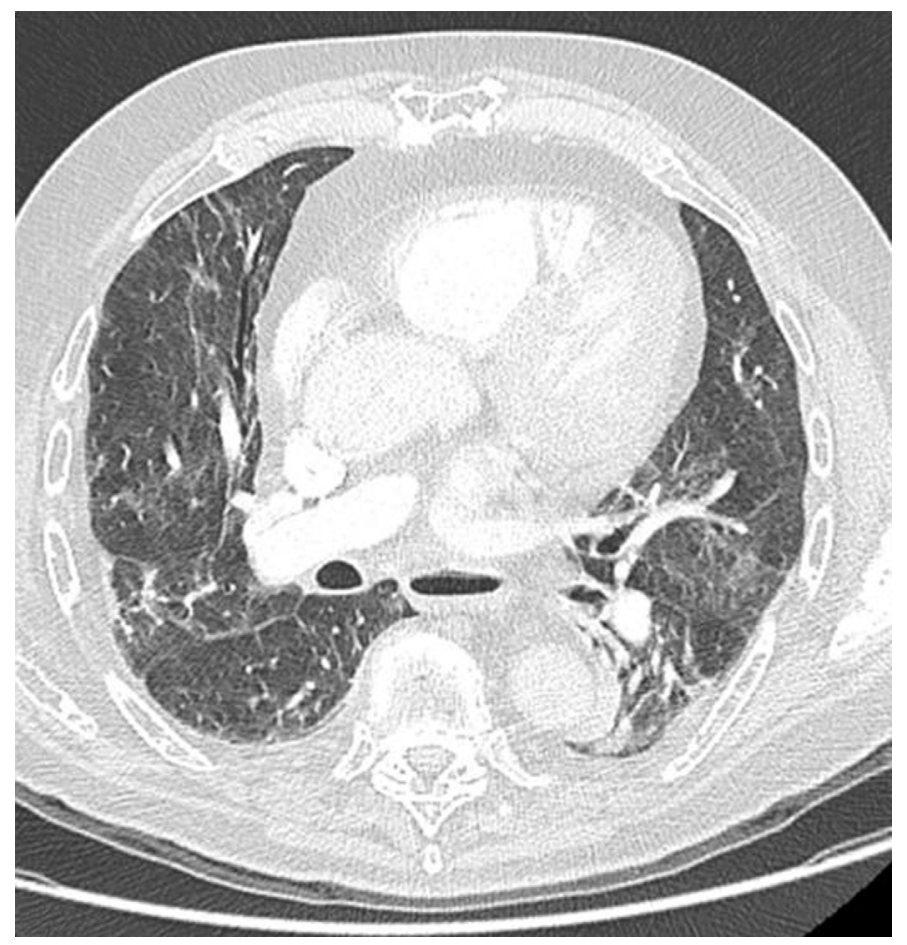

FIGURE 1. Computed tomography showing bilateral patchy interstitial infiltrates.

evolution under broad-spectrum antibiotics. A diagnosis of pulmonary toxicity of thalidomide could, nevertheless, not be formally ruled out. The patient continued treatment with doxorubicin and dexamethasone, and then received consolidative high-dose melphalan with an autologous stem-cell transplant (ASCT) resulting in a partial response.

Disease progression occurred 19 months after ASCT, and bortezomib and dexamethasone were started, again resulting in a partial response. Bortezomib had to be interrupted because of peripheral neuropathy after four cycles. The disease progressed again 23 months after cessation of bortezomib and the patient was started on lenalidomide $\left(15 \mathrm{mg} \cdot \mathrm{day}^{-1}\right.$ on days $1-$ 21 , every 28 days, adapted to renal function). Dexamethasone was added during the first 2 months of treatment but then withheld because of concerns expressed by the patient regarding potential toxicity. A rapid partial response was obtained and it was decided to continue the same treatment until disease progression. The dose was eventually reduced to $10 \mathrm{mg} \cdot \mathrm{day}^{-1}$ on days 1-21, every 28 days, after 16 cycles because of haematological toxicity (neutropenia).

During the course of his 21st cycle of lenalidomide, the patient developed cough and fever. He was first treated with amoxicillin-clavulanate. Because of worsening symptoms and the apparition of dyspnoea, he was admitted to our hospital. Physical examination revealed polypnoea (32 cycles per minute). Oxygen saturation was $88 \%$ while breathing room air. Auscultation revealed bilateral pulmonary crackles. The white cell count showed 2,800 leukocytes per $\mathrm{mm}^{3}$ with $86 \%$ neutrophils, $8 \%$ lymphocytes and no eosinophils. Blood gas analysis revealed severe hypoxaemia. Repeated blood cultures were sterile; pneumococcus and Legionella urinary antigens were negative. The ECG was without significant changes. Brain-type natriuretic peptide concentration was $515 \mathrm{ng} \cdot \mathrm{L}^{-1}$ (normal range $<100 \mathrm{ng} \cdot \mathrm{L}^{-1}$ ). A chest computed tomography with bolus intravenous contrast agent revealed diffuse patchy opacities (fig. 1).

The patient received empirical broad-spectrum antibiotic treatment with high-dose trimethoprim-sulfamethoxazole (the patient was taking this medication at a lower dose as a prophylaxis against Pneumocystis jirovecii pneumonia), spiramycin and cefotaxime, and lenalidomide was discontinued. The bronchoalveolar lavage fluid contained 500,000 cells $\cdot \mathrm{mL}^{-1}$ with $72 \%$ lymphocytes, $2 \%$ neutrophils, $6 \%$ eosinophils and $20 \%$ macrophages. Flow cytometric analysis showed that the vast majority of lymphocytes were T-cells (96\%) with a CD4/ CD8 ratio of 2.4. No infectious agent was found.

Despite receiving empirical diuretic therapy and supplemental oxygen, the patient's condition worsened and the patient required mechanical ventilation. Without any other therapeutic intervention, the patient's condition then progressively improved, permitting weaning from mechanical ventilation.

The possibility of pulmonary lenalidomide toxicity having arisen, this medication was temporarily stopped for a period of 5 months, during which the patient received no other therapy for his myeloma. The disease showed signs of progression after 5 months, thus we decided to start him again on lenalidomide $10 \mathrm{mg} \cdot$ day $^{-1}$ under close medical observation in the hospital. The patient was informed of the potential risks and benefits, and gave his consent. After having received only two doses of $10 \mathrm{mg}$ lenalidomide, he again developed fever, cough and hypoxaemia with bilateral pulmonary infiltrates on the chest radiograph. Lenalidomide was definitively stopped and the patient's condition quickly improved.

The myeloma was treated with three cycles of bendamustine and prednisone without efficacy, then with a combination of melphalan, cyclophosphamide and dexamethasone, which did not control the disease. The patient ultimately died from uncontrolled multiple myeloma, 7 yrs after the initial diagnosis.

A white female was diagnosed with $\lambda$ light chain multiple myeloma at age 82 yrs. The patient was initially treated with high-dose steroids, and then received melphalan, prednisone and thalidomide. Thalidomide had to be interrupted after only 2 months because of neurological toxicity (tremor). The patient received a total of 12 courses of melphalan and prednisone, and went into partial remission. After 4 months without any specific therapy, the disease relapsed and the patient was treated with lenalidomide (10 $\mathrm{mg} \cdot \mathrm{day}^{-1}$ on days $1-21$, then every 28 days) and dexamethasone $\left(20 \mathrm{mg} \cdot \mathrm{week}^{-1}\right)$. She again went into partial remission and received a total of 17 cycles of treatment.

During the course of her 17th cycle, 3 weeks before hospital admission, she began to present a progressively worsening dyspnoea associated with cough, expectoration and fever. She received broad-spectrum oral antibiotics (amoxicillin-clavulanate and levofloxacin) without significant improvement. She finally had to be admitted to the emergency department of our hospital. On physical examination, the patient was conscious, had polypnoea (30 cycles per minute) and had an oxygen saturation of $91 \%$ while breathing room air. Auscultation revealed symmetric bilateral sibilant rales. The white cell count 
showed 8,100 leukocytes per $\mathrm{mm}^{3}$ : $84 \%$ neutrophils, $6 \%$ lymphocytes and no eosinophils. Blood gas analysis revealed mild hypoxaemia with hyperventilation. Repeated blood cultures were sterile; pneumococcus and Legionella urinary antigens were negative. The ECG was without significant changes except tachycardia. A chest computed tomography without intravenous contrast agent revealed diffuse groundglass opacities and consolidation of the left apex.

Lenalidomide was discontinued and a bronchoalveolar lavage was performed before the addition of any antibiotics. The bronchoalveolar lavage fluid contained 360,000 cells $\cdot \mathrm{mL}^{-1}$, with $54 \%$ lymphocytes, $17 \%$ neutrophils, $6 \%$ eosinophils and $25 \%$ macrophages. Flow cytometric analysis showed that the vast majority of lymphocytes were T-cells (97.8\%), with a CD4/ CD8 ratio of 0.66 . Again, no infectious agent was found. Following bronchial endoscopy, the patient's condition worsened and she had to be admitted to the intensive care unit. She required noninvasive ventilatory support and spiramycin was started under the hypothesis of atypical pneumonia. Serology for chlamydiae, Legionella and Mycoplasma were later obtained, and were negative. Lenalidomide was definitively discontinued. The patient's condition progressively improved without mechanical ventilation. She is currently under treatment with bortezomib and dexamethasone for her myeloma.

Iatrogenic drug-induced pneumonias are an increasingly frequent cause of morbidity and mortality. More than 350 medications have already been implicated [1]. Pulmonary toxicity of lenalidomide seems fairly reasonable in our cases based on the exclusion of alternative causes, imaging and bronchoalveolar lavage findings, and clinical course (regression after cessation of lenalidomide in both patients and, in one case, relapse upon taking lenalidomide again).

To the best of our knowledge, five other cases of lenalidomideassociated pulmonary toxicity have been reported, all in patients with multiple myeloma [2-6]. In all these cases, the first symptoms of pulmonary toxicity appeared after a limited treatment duration (2-9 weeks), contrasting with our two patients, who developed the first symptoms after 24 and 17 months, respectively. Pulmonary toxicity has been reported in patients treated with thalidomide $[1,7]$ and more recently with the novel immunomodulatory drug pomalidomide [7]. In none of the cases reported to date did the first signs of pulmonary toxicity appear $>120$ days after first prescription of the drug [7].

Rechallenge in case of suspected drug-induced lung toxicity is potentially harmful to the patient, as illustrated in our first case. It should only be proposed to those patients for whom the expected benefits of the suspected drug(s) are superior to the predictable toxicity and after careful discussion. By no means should it be recommended as a way to confirm a suspected diagnosis of drug-induced lung toxicity.
Pulmonary events are not exceptional in patients with multiple myeloma. Interestingly, in the MM 009 Study, which compared lenalidomide plus dexamethasone with dexamethasone alone in patients with relapsed or refractory myeloma, grade 3-4 pneumonia was reported in $12.4 \%$ of patients treated with lenalidomide plus dexamethasone versus $7.4 \%$ in the dexamethasone-treated group [8].

Physicians should be informed of this possible complication of lenalidomide therapy and evoke the diagnosis whenever a lenalidomide-treated patient presenting with pulmonary infiltrates, fever and hypoxaemia fails to improve after treatment with broad-spectrum antibiotics.

\section{Kamel Amraoui*, Karim Belhadj*, Bernard Maître", Caroline Jannière-Nartey ${ }^{\#}$ and Jehan Dupuis*}

*Lymphoid Malignancies Unit, Assistance Publique - Hôpitaux de Paris, Henri Mondor University Hospital. "Pneumology Unit, Assistance Publique - Hôpitaux de Paris, Henri Mondor University Hospital, Créteil, France.

Correspondence: J. Dupuis, Unité Hémopathies Lymphoïdes, CHU Henri Mondor, 51 Avenue du Maréchal de Lattre de Tassigny, 94010 Créteil Cedex, France. E-mail: jehan.dupuis@hmn. aphp.fr

Statement of Interest: J. Dupuis has received reimbursement for attending a symposium from Celgene.

Provenance: Submitted article, peer reviewed.

\section{REFERENCES}

1 Camus, P. The Drug-Induced Respiratory Disease Website. Pneumotox Online. Version 287. www.pneumotox.com Date last accessed: July 2011.

2 Thornburg A, Abonour R, Smith $\mathrm{P}$, et al. Hypersensitivity pneumonitis-like syndrome associated with the use of lenalidomide. Chest 2007; 131: 1572-1574.

3 Lerch E, Györik S, Feilchenfeldt J, et al. A case of lenalidomideinduced hypersensitivity pneumonitis. Onkologe 2010; 33: 249-252.

4 Chen Y, Kiatsimkul P, Nugent K, et al. Lenalidomide-induced interstitial lung disease. Pharmacotherapy 2010; 30: 325.

5 Coates S, Barker A, Spurgeon S. Reversible pulmonary toxicity due to lenalidomide. J Oncol Pharm Pract 2012; 18: 284-286.

6 Iino M. Interstitial pneumonitis associated with the immunomodulatory drugs thalidomide and lenalidomide. Int J Hematol 2012; 95: 223-224.

7 Geyer H, Viggiano R, Lacy M, et al. Acute lung toxicity related to pomalidomide. Chest 2011; 140: 529-533.

8 Weber DM, Chen C, Niesvizky R, et al. Lenalidomide plus dexamethasone for relapsed multiple myeloma in North America. N Engl J Med 2007; 357: 2133-2142.

DOI: $10.1183 / 09059180.00001912$ 